\title{
Antiga de Revisäa
}

\section{FREQŨÊNCIA CARDÍACA E RISCO CARDIOVASCULAR}

Luiz Antonio Machado César

Trabalho realizado na Unidade Clínica de Coronariopatia Crônica do Instituto do Coração (InCor) do HC - FMUSP

\section{RESUMO}

A freqüência cardíaca ( $F C$ ) também está no centro das determinantes do tempo de vida dos seres vivos animais. Há quase uma constante quando se multiplica a FC pelo número de anos que, em média, vive um animal. O ser humano também

\section{*Correspondência}

Unidade Clínica de Coronariopatia Crônica do Instituto do Coração

(InCor) do HC - FMUSP

Av. Dr. Enéas de Carvalho Aguiar,

44. CEP-05403-000

São Paulo, SP.

Tel: (1 1) 30695387

Fax: (1 1) 30695348 tem correlações interessantes de tempo de vida e FC, com estudos epidemiológicos demonstrando maior sobrevivência naqueles com menor FC em repouso, hipertensos, com doença coronariana e na população geral. Neste artigo, revemos estes estudos epidemiológicos e a perspectiva de se, de fato reduzindo a FC, poderemos aumentar o tempo de vida, no caso dos pacientes com doença cardiovascular, de forma independente de outros fatores.

UniteRmos: Freqüência cardíaca. Doença coronária. Hipertensão arterial. Doença cerebro-vascular. Isquemia miocárdica.

\section{INTRODUÇÃO}

É interessante como a freqüência cardíaca (FC) influencia nossas vidas, provavelmente devido, também, à sua relação com a estimulação adrenérgica, a ponto de ser um fator independente de chance de morte em humanos. No mundo animal, por uma questão biofísica, quanto menor a área corporal, proporcionalmente maior é o gasto energético do organismo para que possa manter a temperatura (pelo menos é o que ocorre com animais poiquilotérmicos). Em conseqüência, quanto menor o animal, maior é sua $\mathrm{FC}$, o que se relaciona de forma inversa com o seu tempo de vida. Em outras palavras, será que temos um "número limite de batimentos cardíacos" para toda uma vida? Se observarmos - que acontece com os mamíferos, parece muito plausível esta possibilidade. Levine avaliou esta premissa e concluiu que o número total de batimentos cardíacos durante a vida é bastante próximo entre todas as espécies de animais avaliadas. Do camundongo à baleia, a multiplicação do número de anos esperados para viver pela $\mathrm{FC}$ resulta sempre em um número muito parecido (Figura I). Esta observação não vale para os humanos, ou seja, nós temos uma expectativa de vida maior, em considerando a nossa FC, caso tracemos um paralelismo com os outros animais. Provavelmente, isto se deve à melhoria das condições de saúde, ao saneamento básico nas cidades, à detecção de doenças, à possibilidade de podermos tratar infecções e a tudo mais que veio com o nosso desenvolvimento intelectual. Por outro lado, quando se observa o que acontece com a expectativa de vida dos humanos e a FC, também verificamos uma relação inversa. Incrível imaginar que, reduzindo a FC de 70 bpm para 60 bpm, em média, pode-se propiciar um aumento da expectativa de vida de 80 anos para acima de 93 anos.

As informações que temos sobre o valor da FC em predizer eventos são várias. Os estudos mostram, tanto na população geral como naqueles já com doença cardiovascular e de forma razoavel-

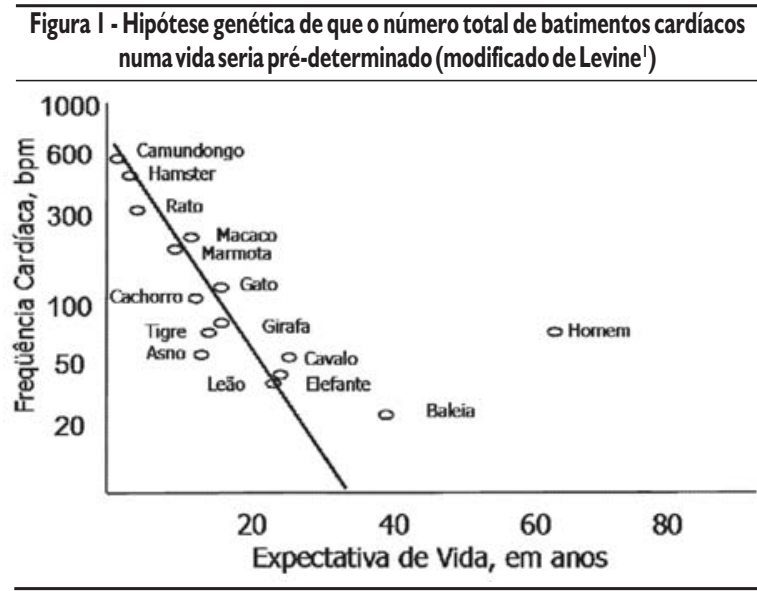

mente consistente, uma correlação direta entre $\mathrm{FC}$ e maior risco de morte. ${ }^{2-6} \mathrm{Em}$ várias situações, nas quais o tratamento inclui a redução da FC, mostra-se a conseqüente redução das mortes e da incidência de infarto do miocárdio. Em especial, isto valeria para os pacientes já com doença coronária, pois a FC é fator determinante do consumo de oxigênio pelo miocárdio. Este é o caso dos estudos com beta-bloqueadores e antagonistas de cálcio que reduzem a $\mathrm{FC}^{7}$

${ }^{10}$. Considerando estes últimos estudos citados, há relação direta e consistente de um menor número de mortes e de infartos do miocárdio quanto maior foi a redução da FC". Desde que a redução da FC parece ser um fator importante, em especial quando se analisam os estudos com beta-bloqueadores, é de se supor o interesse no sentido de tentar entender e até a procurar por fármacos que reduzam a FC, mesmo sem serem bloqueadores beta ou dos canais de cálcio. Esta foi, se não a única, a principal razão 


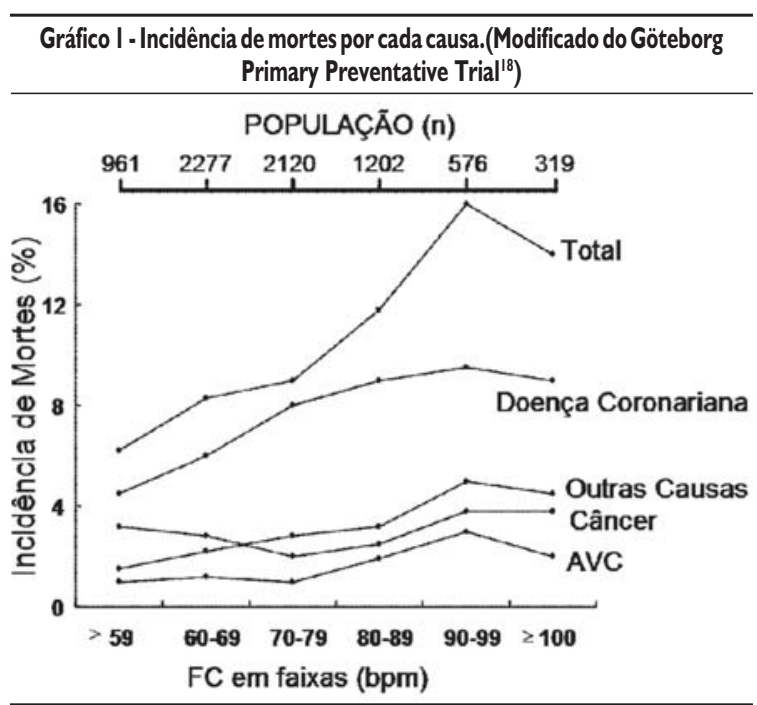

Figura 2 - Mortalidade e suas causas em homens, no estudo de Framingham, conforme a freqüência cardíaca. (Modificado de KannelW. ${ }^{3}$ )

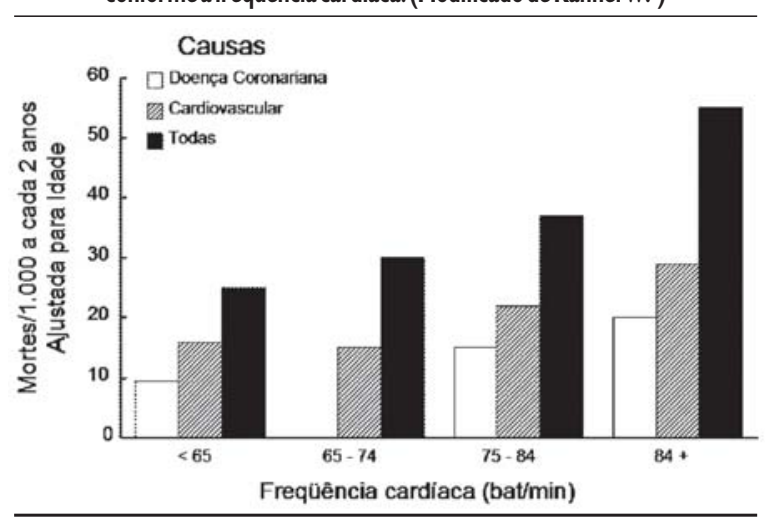

para procurar entender os mecanismos que controlam a despolarização do nó sinusal e, depois, o desenvolvimento de agentes que pudessem interferir nestes mecanismos ${ }^{12}$.

Fisiopatologia - Embora não haja dúvidas sobre a associação entre FC elevada e maior risco de eventos e mortes de causa cardiovascular, não se tem completa certeza de todos os mecanismos envolvidos nesse efeito. Do inadequado preparo físico até um estado geral de saúde ruim são responsabilizados por esse fato, mas não há dúvidas de que a ativação do sistema nervoso autônomo simpático ou mesmo o menor tônus do parassimpático tenham muito a ver com essas observações ${ }^{13,14}$; sendo esses fatos confirmados pelos efeitos que têm os beta-bloqueadores como protetores na isquemia miocárdica. Mas esta não parece ser a única razão, pois em experimentos com um tipo de macaco, da espécie cynomolgus, a ablação do nó sinusal protege estes animais do desenvolvimento da aterosclerose induzida por dieta rica em colesterol, quando comparados aos animais controles ${ }^{15}$. Ou seja, neste modelo, independentemente dos níveis de estimulação adrenérgica, a FC foi determinante de maior indução de aterosclerose. Sendo este um modelo animal, seria necessária alguma observação em humanos que pudesse apontar para a mesma direção. Alguns dados apontam para a mesma direção, como a comunicação da ocorrência de maior gravidade da doença coronariana naqueles com maior valor da mínima FC observada, em jovens após infarto do miocárdio, estudados com monitoração eletrocardiográfica de 24 horas pelo sistema Holter ${ }^{16}$. Os mesmos autores observaram, tempos depois, que, após o infarto do miocárdio, a progressão da doença coronária também se fazia de forma mais intensa, naqueles jovens com maiores valores de $\mathrm{FC}^{17}$. Observações epidemiológicas - São vários os estudos que avaliaram eventos correlacionados com a FC. Vamos destacar alguns destes, em especial os que relacionaram a FC com mortalidade e com morbidade cardiovascular.

Doença coronariana - $O$ estudo que tem o maior tempo de seguimento de pessoas é o de Framingham ${ }^{3}$, com observações que se iniciaram em 1948. Quanto à FC, as observações foram derivadas de uma população alvo de 5.070 indivíduos. Dentre os vários dados que foram tabulados, a FC, avaliada no ECG de repouso a cada dois anos, era um deles. Após 30 anos de seguimento, houve 1.876 mortes, das quais 894 de causas cardiovasculares. Conforme as várias faixas de FC observadas, houve aumento progressivo, tanto em homens (Figura 2) quanto em mulheres, de todas as mortes $(p<0,05)$ desde as por causas cardiovasculares, por infarto do miocárdio e por mortes súbitas $(p<0,00 I)$ relacionadas com maiores valores da FC. Não foi possível estabelecer nenhum valor de corte para a FC a partir do qual haveria maior risco de morrer, pois o efeito da FC foi contínuo. No estudo NHANES I, ${ }^{4}$ a FC foi avaliada numa população com idades de 25 a 74 anos, que tiveram sua inclusão no estudo entre os anos de 1971 e 1974. Estes foram seguidos por um período de 6 a 13 anos, com média de 9,9 anos para os de etnia branca, e 10,3 anos para os de etnia afro-americana. A FC foi avaliada por médico, pela palpação do pulso radial por 30 segundos. $O$ risco relativo de aparecer doença coronariana foi maior naqueles com FC> 84 bpm, quando comparados aos com $\mathrm{FC}<74$ bpm, após ser feita correção para todos os fatores de risco. O mesmo foi observado para as mortes por qualquer causa, ou seja, por causa cardiovascular e não cardiovascular, sendo independentes de outros fatores. Isto foi válido para ambas as etnias e para ambos os sexos, mas a relação foi muito mais intensa para as mulheres de etnia afro-americana. Considerando agora dados da Europa, no estudo de Göteborg (Göteborg Primary Prevention Trial), ${ }^{18} 7.455$ indivíduos foram seguidos por 11,8 anos, em média. A relação entre $F C$ e mortes (classificadas como total, por doença coronariana, por acidente vascular cerebral e por câncer) pode ser vista no Gráfico I. É marcante o progressivo aumento das mortes por causas coronarianas, acidente vascular cerebral e 0 total das correlacionadas com a FC. Isto ficou ainda mais evidente com FC> 
84 bpm, tal qual no estudo NHANES I. Outros três estudos epidemiológicos, de companhias americanas de Chicago que seguiram seus funcionários (considerando a faixa etária de 40 a 64 anos), em análise univariada também mostraram o mesmo efeito de aumento de mortes totais, cardiovasculares e não cardiovasculares de acordo com o aumento da FC. ${ }^{2}$ Mesmo se ajustado para outros fatores de risco cardiovascular, ainda permaneceu o efeito em relação à morte súbita de origem coronariana. Infarto agudo do miocárdio - A FC na admissão de paciente com infarto agudo do miocárdio prevê morte por qualquer causa." Há estreita correlação com os valores da $\mathrm{FC}$, sendo que valores > 110 bpm implicam em risco de quase $50 \%$ de morte, enquanto valores < 69 bpm associam-se com 15\% de mortes. Mais recentemente, Berton et al. ${ }^{19}$ também demonstraram essa relação, mas só em homens e para $\mathrm{FC}>80$ bpm. O mesmo foi observado em relação ao tamanho do infarto, embora esses efeitos pareçam depender, em grande parte, da estimulação adrenérgica, que, entre outros fatores, também depende do tamanho do infarto. ${ }^{20}$ Mas é interessante lembrar que há estudos sugerindo a maior possibilidade de ruptura da placa aterosclerótica em indivíduos com maiores valores de FC. Um desses estudos é o de Heiland et al. ${ }^{21}$, que mostra uma razão de chance muito maior de haver ruptura de placa aterosclerótica em artéria coronária de indivíduos com infarto agudo e, na chegada ao hospital, com FC> 80 bpm.

Hipertensão arterial sistêmica - Há evidências de que hipertensos têm FC maior do que não hipertensos. ${ }^{5}$. Nesse estudo de Framingham, 4.530 indivíduos, com PA $\geq 140 \times 90 \mathrm{mmHg}$ e com idades entre 35 a 74 anos, foram seguidos por 36 anos. Aquele grupo de indivíduos que teve acréscimo na sua FC de 40 bpm ou mais, durante o seguimento, teve mais do que o dobro de mortes. Não havia razões outras, nem doenças, para explicar este aumento da FC nos indivíduos. Desta forma, se confirma o mesmo valor preditor da FC quanto à ocorrência de mortes em pacientes com pressão arterial elevada, tal qual se demonstra para a doença coronariana aguda ou crônica.

Idosos - Há um estudo epidemiológico com I.31। idosos, com idades entre 60 e 100 anos e média de 81 anos, que tinham HAS ou alguma alteração cardiovascular e estavam em ritmo sinusal. Nestes pacientes, a FC foi avaliada por meio do ECG - 24h, sistema Holter e, além da idade e do sexo masculino, a FC também foi fator independente para evento coronariano com aumento da chance de I, I4 para cada aumento de cinco BPMs na FC, após controle da interferência dos outros fatores de risco ${ }^{22}$.

De todas as associações avaliadas, sem dúvida é muito forte aquela da $\mathrm{FC}$ relacionada à morte súbita. Embora também tenham informações suficientes relacionando a maior FC com a mortalidade cardiovascular e total, esta associação não é uniforme nos estudos. Ou seja, quando se corrige para múltiplos fatores de risco, a associação já não permanece em mulheres e, em alguns dos estudos, nem em homens, pelo menos para todas as causas de morte. Mas com doença coronária e, principalmente, com morte súbita permanece a associação. $O$ que é surpreendente é a associação da maior FC com mortes não cardiovasculares, caso dos estudos de Chicago $^{2}$ e de Framingham ${ }^{3}$. Desta forma, parece bastante evidente a associação da FC com mortalidade nos casos de pacientes com risco de doença cardiovascular e naqueles que sabidamente já a tem. Ao mesmo tempo, fica evidenciada a necessidade de mais estudos, com o intuito de entender os mecanismos que possam explicar esses achados e até a sua dependência com a atividade física, a hipertrofia ventricular esquerda, a resistência à insulina, além de outros mecanismos neuro-humorais que podem estar mascarando esses resultados. E a dúvida final: será que a redução da FC por si só, sem o efeito dos fármacos atuais, mas com a utilização de outros que interfiram em outros mecanismos, pode aumentar nossa expectativa de vida?

\section{Conflito de interesse: não há.}

\section{SUMMARY}

\section{Heart Rate and CaRdiovascular RISK}

Heart rate $(H R)$ is known to be one of the factors that in the animal world are related to mortality. When one multiplies resting $H R$ by the average life time, for each animal species, the result is almost the same number, similar to a constant. Apparently, each class of living beings has its "maximal number of heart beats for a life". Herein considerations are made about the relationship between HR and survival in human beings. Some epidemiological studies are reviewed, especially those related to cardiovascular and coronary diseases correlated to deaths and discussions are directed towards the perspective of living longer by lowering the HR. [Rev Assoc Med Bras 2007; 53(5): 456-9]

KEY wORDS: Heart rate. Coronary diseases. Arterial Hypertension. Cerebrovascular disease. Myocardial ischemia.

\section{REFERÊNCIAS}

I. Levine HJ. Rest heart rate and life expectancy. J Am Coll Cardiol. 1997:30: 1 104-6.

2. Dyer AR, Persky V, Stamler J, Shekelle RB, Berkson DM, Lepper M, et al. Heart rate as a prognostic factor for coronary heart disease and mortality: findings in three Chicago epidemiologic studies. Am J Epidemiol. | 1980; | | 2:736-49.

3. Kannel W, Kannel C, Paffenbarger R, Cupples A. Heart rate and cardiovascular mortality: The Framingham study. Am Heart J. 1987:1| 3:1489-94.

4. Gillum R, Makuc D, Feldman J. Pulse rate, coronary heart disease, and death: The NHANES I epidemiologic follow-up study. Am Heart J. 1991; $121: 172-7$

5. Gillman M, Kannel W, Belanger A, D'Agostino R. Influence of heart rate on mortality among persons with hypertension: The Framingham study. Am Heart J. 1993; 125:1 |48-54.

6. Goldberg R, Larson M, Levy D. Factors associated with survival to 75 years of age in middle-aged men and women: The Framingham study. Arch Intern Med. 1996;156:505-9.

7. The Norwegian Multicenter Study Group. Timolol-induced reduction on mortality and reinfarction in patients surviving acute myocardial infarction. N Engl J Med. 1981;304:801-7.

8. Beta-Blocker Heart Attack Trial Research Group. A randomized trial of propranolol in patients with acute myocadial infarction. I. Mortality results. JAMA. 1982;247:1707-14.

9. The Multicenter Diltiazem Postinfarction Trial Research Group. Effect of diltiazem on mortality and reinfarction after myocardial infarction. 
N Engl J Med. 1988;319:385-9.

10. The Danish Study Group on Verapamil in Myocardial Infarction. Effect of verapamil on mortality and major events after acute myocardial infarction (The Danish Verapamil Infarction Trial II - DAVIT-II). Am J Cardiol. 1990;66:779-85.

I I. Hjalmarson A, Gilpin E, Kjeksus J, Schieman G, Nicod P, Henning H, Ross J. Influence of heart rate on mortality after acute myocardial infarction. Am J Cardiol. 1990;65:547-53.

I 2. Monnet X, Ghaleh B, Colin P, De Curzon PO, Giudicelli JF, Berdeaux A. Effects of heart rate reduction with ivabradine on exercise-induced myocardial ischemia and stunning. J Pharmacol Exp Ther 2001;299: I133-9

13. Kjekshus ]. Commets: beta-blockers: heart rate reduction a mechanism of benefit. Eur Heart J. 1985;6:29-30.

14. Singh BN. The relevance of sympathetic activity in the pharmacological treatment of chronic stable angina. Can J Cardiol. 1999; I5(Suppl A): | 5A-2|A.

I 5. Beere P, Glagov S, Zarins $C$. Retarding effect of lowered heart rate on coronary atherosclerosis. Science. 1984:226:180-2

16. Perski A, Hamsten A, Lindvall K, Theorell T. Heart rate correlates with severity of coronary atherosclerosis in young postinfarction patients. Am Heart J. 1988;116:1369-73.

17. Perski A, Ollson G, Lando C, Faire U, Theorell T, Hamsten A. Minimum heart rate and coronary atherosclerosis: Independent relations to global severity and rate of progression of angiographic lesions in men with myocardial infarction at a young age. Am Heart J. 1992; 1 23:609-16.

18. Wilhelmsen L, Berglund G, Elmfeldt $D$ et al. The multifactor primary prevention trial in Göteborg, Sweden. Eur Heart J. 1986;7:279-88.
19. Berton GS, Cordiano R, Palmieri R, Gheno G, Mormino P, Palatini P. Heart rate during myocardial infarction: relationship with one-year global mortality in men and women. Can J Cardiol. 2002; | 8:495-502.

20. Shell W, Sobel B. Deleterious effects of increased heart rate on infarct size in the conscious dog. Am J Cardiol. 1973;31:474-9.

21. Heidland EU, Strauer BE. Left ventricular muscle mass and elevated heart rate are associated with coronary plaque disruption. Circulation. 2001; 1 04:1 477-82

22. Aronow W. Ahn C. Mercando A, Epstein S. Association of average heart rate on 240hour ambulatory electrocardiograms with incidence of new coronary events at 48-month follow-up in 1,31। patients (mean age 81 years) with heart disease and sinus rhythm. Am J Cardiol. 1996;78: 1 175-6. 